\title{
The Final Stage of Gravitationally Collapsed Thick Matter Layers
}

\author{
Piero Nicolini, ${ }^{1}$ Alessio Orlandi, ${ }^{2}$ and Euro Spallucci ${ }^{3}$ \\ ${ }^{1}$ Frankfurt Institute for Advanced Studies (FIAS) and Institute for Theoretical Physics, Johann Wolfgang Goethe University, \\ D-60438 Frankfurt am Main, Germany \\ ${ }^{2}$ Dipartimento di Fisica, Università di Bologna and INFN, I-40126 Bologna, Italy \\ ${ }^{3}$ Dipartimento di Fisica, Università di Trieste and INFN, I-34151 Trieste, Italy
}

Correspondence should be addressed to Alessio Orlandi; alessio.j.orlandi@gmail.com

Received 17 September 2013; Accepted 8 November 2013

Academic Editor: Emil Akhmedov

Copyright ( $\odot 2013$ Piero Nicolini et al. This is an open access article distributed under the Creative Commons Attribution License, which permits unrestricted use, distribution, and reproduction in any medium, provided the original work is properly cited.

In the presence of a minimal length, physical objects cannot collapse to an infinite density, singular, matter point. In this paper, we consider the possible final stage of the gravitational collapse of "thick" matter layers. The energy momentum tensor we choose to model these shell-like objects is a proper modification of the source for "noncommutative geometry inspired," regular black holes. By using higher momenta of Gaussian distribution to localize matter at finite distance from the origin, we obtain new solutions of the Einstein equation which smoothly interpolates between Minkowski's geometry near the center of the shell and Schwarzschild's spacetime far away from the matter layer. The metric is curvature singularity free. Black hole type solutions exist only for "heavy" shells; that is, $M \geq M_{e}$, where $M_{e}$ is the mass of the extremal configuration. We determine the Hawking temperature and a modified area law taking into account the extended nature of the source.

\section{Introduction}

Relativistic, self-gravitating matter shells have been thoroughly investigated in different sectors of theoretical physics: "...from cosmic inflation to hadronic bags" [1]. Remarkable applications of gravitational shell models can be found in the framework of inflationary cosmology, where both the "birth" and the evolution of vacuum bubbles can be effectively described in terms of the dynamics of the boundary surface engulfing a false vacuum domain [1-4]. Matter shells in general relativity are modeled as "zero-thickness" membranes endowed with some characteristic tension determined by the underlying classical, or quantum, physics. Neglecting the real width of the mass-energy distribution affects Einstein's equations by introducing a surface of discontinuity in the background spacetime. This approximation allows to encode all the dynamics in the matching condition between the inner and outer geometries $[5,6]$. Furthermore, contracting matter shells provide useful analytic toy models of collapsing massive bodies leading to black hole formation. In the spherically symmetric case, the only dynamical degree of freedom is given by the shell radius and the system can be quantized according to the standard principle of quantum mechanics.
In this framework, self-gravitating quantum shells open a window over the still "murky" quantum features of evaporating mini black holes [7-11].

In this paper, we are going to investigate the static, final stage of collapsed matter shell in the presence of a fundamental minimal length forbidding the shell to contract into a singular matter point. The emergence of a minimal length, as a new fundamental constant of nature on the same ground as $c$ and $\hbar$, is a general feature of different approaches to quantum gravity [12-14].

In recent years, we showed that the very concept of "point particle" is meaningless if there exists a lower bound to physically measurable lengths. For instance, in a series of papers the repercussions of a natural ultraviolet cutoff have been analyzed in the context of quantum field theory [15-20]. This basic notion can be also encoded into the Einstein equations through a proper choice of the energy momentum tensor. The most remarkable outcomes of this procedure are the disappearance of curvature singularities in the solutions of the Einstein equations [21], a regular behavior of the Hawking temperature which allows to determine the physical character of the evaporation remnant [22-26], and a different form of the relation between entropy and area of 
the event horizon reproducing the celebrated area law for large, semiclassical black holes [27, 28].

The procedure has been applied in the whole array of physically meaningful black hole solutions like the neutral, nonrotating case [29-32], the charged, nonrotating case [33, 34], the "dirty," neutral, and nonrotating case [35], and the spinning, neutral [36] and charged cases [37]. In addition, the regularity of the above metrics has been exploited in several complementary contexts like the decay of the de Sitter universe by quantum black hole nucleation [38] and the case of dimensionally reduced spacetimes [39]. The procedure we followed has been also recognized as a special result [40] of recently formulated nonlocal gravity proposals [41, 42] with important cross-fertilization in the AdS/CFT paradigm [43].

The paper is organized as follows. In Section 2, we will derive new solutions of Einstein's equations describing spherically symmetric, static, self-gravitating, thick matter layers in the presence of a fundamental minimal length. These types of objects are modeled by means of a proper modification of the source for "noncommutative geometry inspired," regular black holes. We replace the Gaussian profile of mass-energy, peaked around the origin, with higher moments of Gaussian distribution with maxima shifted at finite distance from the origin. The finite width of the distribution is determined by the minimal length. In the case of lump-type objects, the minimal length measures the spread of mass-energy around the origin and removes the curvature singularity. For finite width matter layers, with energy density vanishing at short distance, not only the central curvature singularity is removed, but the extrinsic curvature discontinuity between "inner" and "outer" geometry is cured as well. Spacetime geometry is continuous and differentiable everywhere and smoothly interpolates between Minkowski's metric near the center and Schwarzschild's spacetime far away from the matter layer.

As in the cases previously discussed, black hole type solutions exist only for "heavy" shells; that is, $M \geq M_{e}$, where $M_{e}$ is the mass of the extremal configuration. "Light" matter layers with $M<M_{e}$ will settle down in a smooth solitonic type configuration with no horizons or curvature singularity.

In Section 3, we study the thermodynamic properties of black hole solutions and determine both the Hawking temperature, $T_{H}$, and the relation between entropy and area of the horizon. We recover the celebrated area law in the limit of large, semiclassical black holes. On a general ground, we find the leading term is one-fourth of the area but in units of an "effective" gravitational coupling constant, $G_{N}\left(r_{+}\right)$, depending on the radius of black hole. For "large" $r_{+}$, the Newton constant is recovered. Finally, in Section 4, we will draw the conclusions.

\section{Thick Shells}

In a previous series of papers we solved the Einstein equations including the effects of a minimal length on a proper energymomentum tensor

$$
R_{\mu \nu}-\frac{1}{2} g_{\mu \nu} R=8 \pi G_{N} T_{\mu \nu}
$$

where $T_{\mu \nu}=\operatorname{diag}\left(\rho, p_{r}, p_{\perp}, p_{\perp}\right)$. The energy density is a minimal width Gaussian distribution

$$
\rho=\rho_{0}(r) \equiv \frac{M}{(4 \pi \theta)^{3 / 2}} e^{-r^{2} / 4 \theta},
$$

representing a "blob-like" object, centred around the origin, with a characteristic extension given by $l \propto \sqrt{\theta}$. A simple way to derive the above energy profile is based on the following considerations. At classical level point-like objects in spherical coordinates are described by a profile

$$
\rho_{\mathrm{cl}}(r)=\frac{M}{4 \pi r^{2}} \delta(r)
$$

where $\delta(r)$ is the Dirac delta. We recall that a Dirac delta function can be represented as the derivative of a Heaviside step-function $\Theta(r)$

$$
\delta(r)=\frac{d}{d r} \Theta(r)
$$

However, in the presence of a minimal length the very concept of sharp step is no longer meaningful. Rather we expect the local loss of resolution to sweeten the step. Accordingly, it can be shown that in the framework of a minimal length a modified step function can be defined through an integral representation of the Heaviside function without taking the limit $\sqrt{\theta} \rightarrow 0$ [44]:

$$
\Theta(r) \longrightarrow P_{0}(r)=\frac{1}{\sqrt{4 \pi} \theta^{3 / 2}} \int_{0}^{r} x^{2} e^{-x^{2} / 4 \theta} d x .
$$

The Gaussian profile (2) is therefore obtained as

$$
\rho_{0}(r)=\frac{M}{4 \pi r^{2}} \frac{d}{d r} P_{0}(r) .
$$

In order to solve Einstein's equations, we need to determine the remaining components of the energy-momentum tensor. The radial pressure $p_{r}$ is fixed by the equation of state $p_{r}=$ $-\rho$ reproducing the de Sitter "vacuum" equation of state at short distance. This is a key feature to build up a regular, stable configuration, where the negative pressure balances the gravitational pull. In other words, the singularity theorem is evaded by a violation of null energy condition triggered by the short distance vacuum fluctuations.

Finally, the tangential pressure $p_{\perp}$ is obtained in terms of $\rho$ by the divergence-free condition $\nabla_{\mu} T^{\mu \nu}=0$.

As the source is static and spherically symmetric, the line element can be cast in the form

$$
d s^{2}=-f(r) d t^{2}+\frac{d r^{2}}{f(r)}+r^{2} \Omega^{2}
$$

with

$$
f(r)=1-\frac{2 G_{N} m(r)}{r} .
$$

The cumulative mass distribution $m(r)$ is given by

$$
m(r)=4 \pi \int_{0}^{r} d r^{\prime}\left(r^{\prime}\right)^{2} \rho_{0}\left(r^{\prime}\right) .
$$




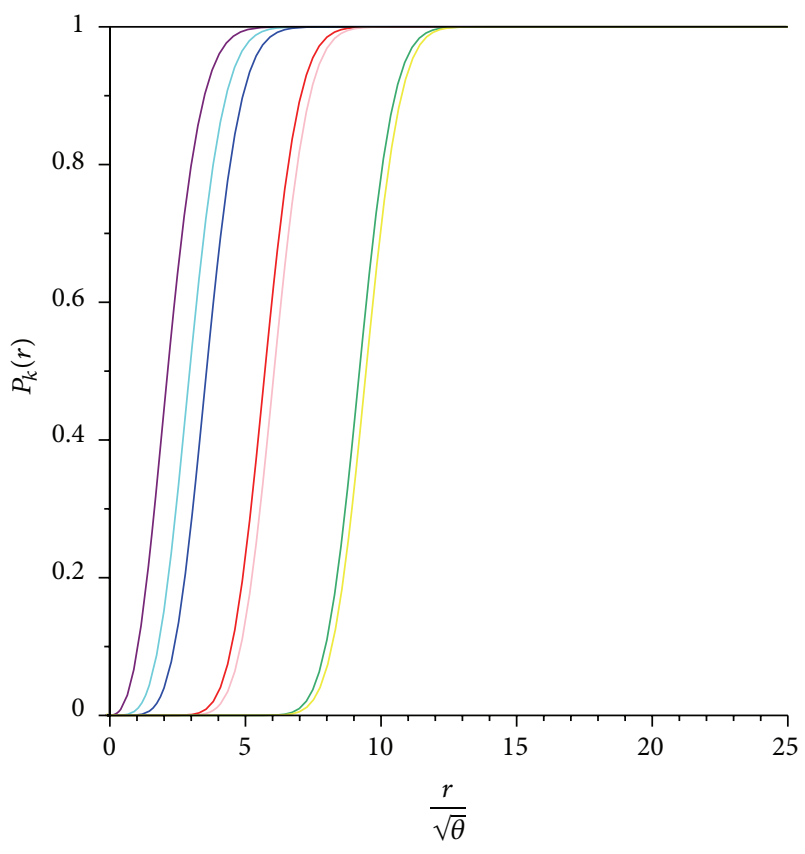

FIgURE 1: Plot of the function $P_{k}(r)$ as a function of $r / \sqrt{\theta}$ for $k=0$ (purple), $k=1$ (cyan), $k=2$ (blue), $k=7$ (red), $k=8$ (pink), $k=20$ (green), and $k=21$ (yellow). For comparison, the Heaviside function is plotted in black.

We notice that the parameter $M$ corresponds to the total mass energy of the system; namely,

$$
M=\lim _{r \rightarrow \infty} m(r) .
$$

The above profile cures the usual Dirac delta (singular) distribution associated to a point particle, and leads to a family of regular black hole solutions [29-39].

A crucial point at the basis of the above line of reasoning is the modification of the step function in the presence of a minimal length. We notice that the profile (5) is just one of the possible choices one can have to account for the loss of resolution of the edge of the step [44]. In other words, there exist alternative representations of the Heaviside function for which one can deliberately avoid the limit $\sqrt{\theta} \rightarrow 0$. For instance, the family of distributions

$$
\Theta(r) \longrightarrow P_{k}(r)=\frac{1}{\sqrt{\pi} \theta^{k+3 / 2}} \frac{(k+1) !}{[2(k+1)] !} \int_{0}^{r} x^{2 k+2} e^{-x^{2} / 4 \theta} d x
$$

account for minimal length effects growing with the index $k$, where $k=0,1,2, \ldots$ is a natural number (see Figure 1). This can be seen by the limit $r / \sqrt{\theta} \gg 1$

$$
P_{k}(r) \approx 1-\frac{4^{k+1}}{\sqrt{\pi}} \frac{(k+1) !}{[2(k+1)] !}\left(\frac{r}{2 \sqrt{\theta}}\right)^{2 k+1} e^{-r^{2} / 4 \theta}
$$

which shows that the distributions $P_{k}(r)$ reach the plateau (i.e., $P_{k} \approx 1$ ) more slowly as $k$ increases.

Here, we want to explore the nature of the solutions emerging for the above admissible profiles for a modified step

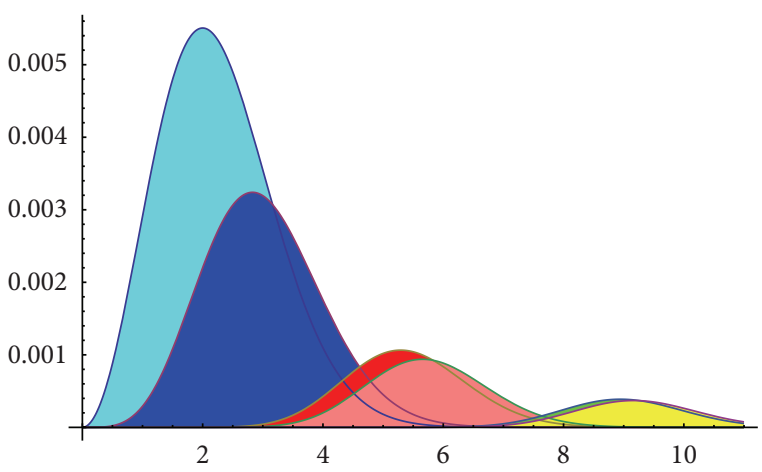

FIGURE 2: Plot of the radial density profile $\rho(r)$ as a function of $r$ in $\sqrt{\theta}$-units for $k=1$ (cyan), $k=2$ (blue), $k=7$ (red), $k=8$ (pink), $k=20$ (green), and $k=21$ (yellow). The function $\rho(r)$ has been normalized so that its integration over a spherical volume gives 1.

function. By replacing $P_{0}$ with a generic $P_{k}$ in (6), we derive the following energy density profile:

$$
\rho_{k}(r) \equiv M \frac{r^{2 k} e^{-r^{2} / 4 \theta}}{4^{k+2} \pi \theta^{k+3 / 2} \Gamma(k+3 / 2)},
$$

which corresponds to higher moments of the Gaussian.

For $k=0$, the function $\rho_{k}$ turns into the Gaussian distribution, centred around the origin, while for $k \geq 1$ the matter distribution is more and more diluted near the origin, being peaked at $r_{M}=2 \sqrt{k \theta}$. As a result, the density function (13) describes a whole family of "mass-degenerate" shells, with the same $M$, but concentrated at a distance given by $r_{M}$ (see Figure 2). Equation (13) discloses further properties of the minimal length, which assumes a new intriguing meaning. In the case of point particle, $\sqrt{\theta}$ represents the spread of the object around the origin. For matter shells, we see that $\sqrt{\theta}$ relates to the shell thickness or, in other words, a measure of the intrinsic fuzziness of the layer. Just as a particle cannot be exactly localized at a single point, we cannot have zero-width layers as well. Thus, as the matter distribution is smooth everywhere, there is no discontinuity in the extrinsic curvature between the "inner" and "outer" geometries. No matching condition is required and we can look for a single, smooth, metric inside, across, and outside the matter layer.

We can define the distance between two shells corresponding to different moments as the distance between the peaks:

$$
\frac{\Delta r_{M}}{2 \sqrt{\theta}}=\sqrt{k+1}-\sqrt{k}
$$

We see that for higher moments $\Delta r_{M}$ vanishes as we are at length scale much larger than $\sqrt{\theta}$ and the relative distance cannot be resolved anymore.

A stable solution of the Einstein equations can be obtained by sourcing the gravitational field by the energy momentum tensor of an anisotropic fluid; the choice $p_{r}=$ $-\rho_{k}$ for the matter equation of state allows to have $g_{00}=-g_{r r}^{-1}$. Furthermore, the hydrodynamic equilibrium equation will 


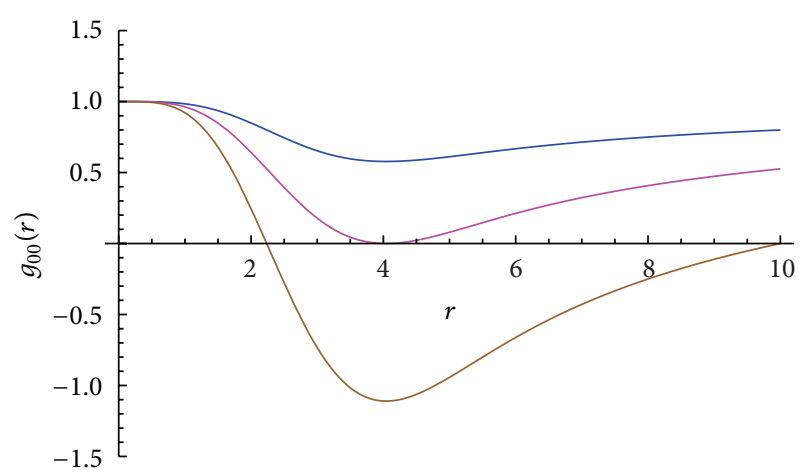

Figure 3: Plot of $g_{00}(r)$ as a function of $r$ in $\sqrt{\theta}$-units for fixed value of $k=1$ and different values of the mass $M$; that is, $M=1$ (blue), $M=M_{e}$ (magenta), and $M=5$ (brown). Here, $M_{e} \simeq 2.36976$ is the extremal mass for the case $k=1$.

give $p_{\perp}$ in terms of $\rho_{k}$, while the metric itself results in being independent from $p_{\perp}$. The solution of the Einstein equations reads in geometric units, $c=1, G_{N}=1$ :

$$
\begin{gathered}
d s^{2}=-\left(1-\frac{2 m(r)}{r}\right) d t^{2}+\left(1-\frac{2 m(r)}{r}\right)^{-1} d r^{2}+r^{2} d \Omega^{2}, \\
m(r) \equiv 4 \pi \int_{0}^{r} d r^{\prime} r^{\prime 2} \rho\left(r^{\prime}\right)=M \frac{\gamma\left(k+3 / 2 ; r^{2} / 4 \theta\right)}{\Gamma(k+3 / 2)},
\end{gathered}
$$

where $\gamma\left(k+3 / 2 ; r^{2} / 4 \theta\right)$ is the lower, incomplete Euler Gamma function. By taking into account the asymptotic form of $\gamma(k+$ $3 / 2 ; r^{2} / 4 \theta$ ) for small argument, one finds that the metric (15) is essentially flat near the origin, the contrary to what happens for the case $k=0$ in which a regular de Sitter core forms. Indeed, one finds that

$$
\begin{aligned}
f(r) \simeq 1- & \frac{1}{2 k+3}\left[\frac{4 M}{r \Gamma(k+3 / 2)}\right]\left(\frac{r^{2}}{4 \theta}\right)^{k+3 / 2}, \\
d s^{2}= & -\left(1-O\left(r^{2 k+2}\right)\right) d t^{2} \\
& +\left(1-O\left(r^{2 k+2}\right)\right)^{-1} d r^{2}+r^{2} d \Omega^{2} .
\end{aligned}
$$

The asymptotic behavior (18) can be seen as a generalization of the "Gauss Theorem": inside an empty, classical, thin shell of matter, the Newtonian gravitational field is zero; that is, spacetime is flat. Introducing a finite width density, smoothly decreasing both toward the origin and space-like infinity, causes small deviations from perfect flatness.

Going back to (15), the presence of event horizons is read from the zeroes of $g_{r r}^{-1}$ (see Figure 3). Due to the nontrivial structure of the metric, it is more convenient to look at the "horizon equation" in the form

$$
M \equiv U\left(r_{H}\right), \quad U\left(r_{H}\right) \equiv \frac{r_{H}}{2} \frac{\Gamma(k+3 / 2)}{\gamma\left(k+3 / 2 ; r_{H}^{2} / 4 \theta\right)} .
$$

The problem of finding the horizons in the metric (15) is mapped in the equivalent problem of determining the turning

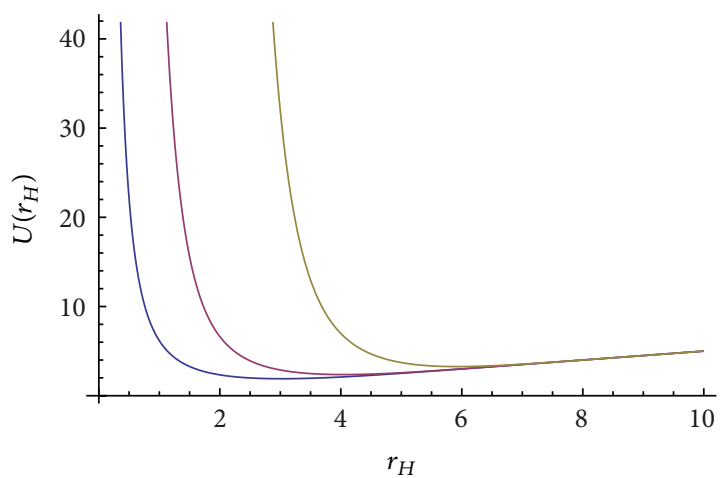

FIGURe 4: Plot of $U\left(r_{H}\right)$ as a function of $r_{H}$ in $\sqrt{\theta}$-units for $k=0$ (blue), $k=1$ (magenta), and $k=4$ (brown).

points for the motion of a "test particle" of energy $M$ subject to the "potential" $U\left(r_{H}\right)$. The intersection(s) between a line $M=$ const. and the plot of the function $U\left(r_{H}\right)$, in the plane $M-r_{H}$, represent the allowed radii of inner/outer event horizons (see Figure 4). The asymptotic behavior of $U\left(r_{H}\right)$ is obtained from the corresponding approximate forms of $\gamma$ :

$$
\begin{gathered}
U\left(r_{H}\right) \approx \frac{r_{H}}{2}, r_{H} \gg \sqrt{\theta}, \\
U\left(r_{H}\right) \approx \sqrt{\theta} \Gamma\left(k+\frac{5}{2}\right)\left(\frac{r_{H}^{2}}{4 \theta}\right)^{-(k+1)}, r_{H} \ll \sqrt{\theta} .
\end{gathered}
$$

Thus, $U\left(r_{H}\right)$ is a convex function with a single minimum determined by the condition

$$
\frac{d U}{d r_{H}}=0 \longrightarrow \frac{r_{e}^{2 k+3}}{2^{2 k+2} \theta^{k+3 / 2}}=\gamma\left(k+\frac{3}{2} ; \frac{r_{e}^{2}}{4 \theta}\right) e^{r_{e}^{2} / 4 \theta} .
$$

The radius $r_{e}$ represents the size of an extremal configuration with a couple of degenerate horizons: $r_{-}=r_{+} \equiv r_{e}$. Once $r_{e}$ is numerically determined from (21), one gets the corresponding mass $M_{e}$ from the potential:

$$
M_{e}=U\left(r_{e}\right)=U_{\min } .
$$

An order of magnitude estimate for $r_{e}$ and $M_{e}$ can be obtained by keeping only the leading theta term:

$$
r_{e} \propto \sqrt{\theta} \longrightarrow M_{e} \propto \frac{\sqrt{\theta}}{L_{\mathrm{Pl}}} M_{\mathrm{Pl} .},
$$

where $L_{\mathrm{Pl}}$ is the Planck length; that is, $L_{\mathrm{Pl} .}=M_{\mathrm{Pl}}^{-1}=\sqrt{G_{N}}$. This estimate suggests that (near/)extremal configurations are close to a full quantum gravity regime. As such, they are appropriate candidates to describe the end-point of the Hawking evaporation process where the semiclassical description breaks down.

In summary

(i) for $M>M_{e}$, we find a geometry with noncoincident inner and outer horizons $r_{-}<r_{+}$. It is worth to remark that there is no curvature singularity in $r=0$. Spacetime is flat near the origin; 
(ii) for $M=M_{e}$, we have an extremal configuration with a pair of degenerate horizons, $r_{-}=r_{+} \equiv r_{e}$.

(iii) for $M<M_{e}$, there are neither horizons nor curvature singularities. The metric is smooth and regular everywhere. The shell is too light and diluted to produce any relevant alteration of the spacetime fabric. After collapse, it will settle into a sort of solitonic object with no horizon of curvature singularity.

Massive layers will produce horizons, but no curvature singularities. This is a crucial point which marks a departure with respect to all the existing literatures. Our approach must not be confused with previous contributions in which generalized matter shells containing polytropic and Chaplygin gas cannot ultimately resolve the emergence of singularities [45].

We can estimate the size of these objects by solving iteratively (19) and truncating the procedure at the first order in the expansion parameter $\exp \left(-M^{2} / \theta\right)$ :

$$
r_{+} \simeq 2 M\left[1-\frac{\left(M^{2} / \theta\right)^{k+1 / 2}}{\Gamma(k+3 / 2)} e^{-M^{2} / \theta}\right] .
$$

This quantity can be compared with the shell mean radius

$$
\langle r\rangle \equiv \frac{4 \pi}{M} \int_{0}^{\infty} d r r^{2} r \rho(r)=2 \sqrt{\theta} \frac{\Gamma(k+2)}{\Gamma(k+3 / 2)} .
$$

We notice that for $k \gg 1$ the leading contribution to the ratio $r_{+} /\langle r\rangle$ is given by

$$
\frac{r_{+}}{\langle r\rangle} \approx \frac{M}{\sqrt{\theta}} \frac{1}{\sqrt{k+1}} .
$$

Thus, for an assigned $M$ the ratio decreases with $k$. The horizon radius is determined by the total mass energy $M$ and is weakly $k$ dependent. On the contrary, $\langle r\rangle \propto \sqrt{k+1} \sqrt{\theta}$ and grows with $k$, which means that for higher moments the horizon is surrounded by a cloud of matter.

\section{Thermodynamics}

Massive shells will collapse into black holes described by the line element (7), (8). This is not the end of story as these objects are semiclassically unstable under Hawking's emission. We are now ready to study the thermodynamic properties of these solutions starting from the Hawking temperature:

$$
T_{H}=\frac{1}{4 \pi r_{+}}\left[1-\frac{r_{+}^{2 k+3}}{2^{2 k+2} \theta^{k+3 / 2}} \frac{e^{-r_{+}^{2} / 4 \theta}}{\gamma\left(k+3 / 2 ; r_{+}^{2} / 4 \theta\right)}\right] .
$$

It can be easily verified that $T_{H}$ is vanishing for the extremal configuration $T_{H}\left(r_{+}=r_{e}\right)=0$. For $k=0$, we obtain the temperature of the black hole as in [31]. The behavior of the temperature can be found in Figure 5 and Table 1. We notice that the regularity of the manifold for any $k$ leads to a cooling down of the horizon in the terminal phase of the Hawking process. At $r_{+}=r_{\max }$, the presence of a maximum

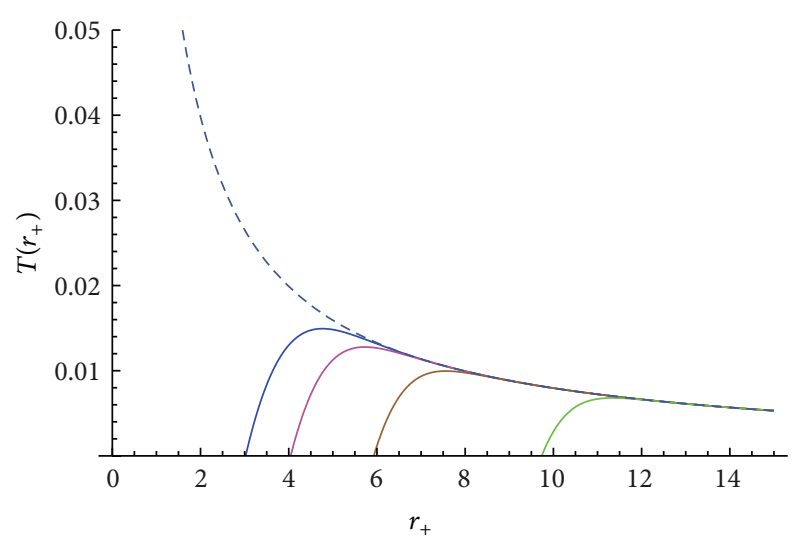

FIGURE 5: Plot of $T\left(r_{+}\right)$as a function of $r_{+}$in $\sqrt{\theta}$-units for $k=0$ (blue), $k=1$ (magenta), $k=4$ (brown), and $k=15$ (green). The dashed line represents the classical case; that is, $\theta=0$.

TABLE 1: Some values of parameters of the matter shells.

\begin{tabular}{lcccc}
\hline & $r_{e}$ & $M_{e}$ & $T_{\max }$ & $r_{\max }$ \\
\hline$k=0$ & 3.0224 & 1.9041 & 0.014937 & 4.76421 \\
$k=1$ & 4.0431 & 2.3698 & 0.012783 & 5.72632 \\
$k=4$ & 5.9269 & 3.2647 & 0.009960 & 7.56069 \\
$k=15$ & 9.7347 & 5.1245 & 0.006799 & 11.3419 \\
\hline
\end{tabular}

temperature corresponds to an infinite discontinuity of the heat capacity which is usually interpreted as the signal of a "change of state" for the system. For $r_{+}>r_{\max }$, the black hole is thermodynamically unstable and increases its temperature by radiating away its own mass. After crossing $r_{\max }$, that is, for $r_{+}<r_{\max }$, the black hole enters a stability phase asymptotically ending into a degenerate (zero-temperature) extremal configuration. By increasing $k$, we just lower down the maximum temperature. As a result, the solution is unaffected by any relevant quantum back reaction as already proved for the case $k=0$ in [31].

A further important consequence of $T_{H} \leq T_{\max }$ is that (in extradimensional models) the black hole mainly radiates on the brane [46], it never becomes hot enough to warm up the bulk in a significant way.

The area-entropy law can be recovered from the relation

$$
d M=T_{H} d S
$$

where $d S$ is the horizon entropy variation triggered by a variation $d M$ in the total mass energy $M$. In order to translate the first law of black hole thermodynamics (28) into a relation involving the area of the event horizon, we need to write mass energy variation as

$$
d M=\frac{\partial U}{\partial r_{+}} d r_{+}
$$

and to take into account that the minimum of $U\left(r_{+}\right)$is the mass of the extremal black hole. Thus, when integrating 
(29), the lower integration limit is the radius of the extremal configuration

$$
\begin{aligned}
S & =\int_{r_{e}}^{r_{+}} d x \frac{1}{T_{H}} \frac{\partial U}{\partial x} \\
& =2 \pi \Gamma\left(k+\frac{3}{2}\right) \int_{r_{e}}^{r_{+}} d r \frac{r}{\gamma\left(k+3 / 2 ; r^{2} / 4 \theta\right)},
\end{aligned}
$$

where we have inserted (27) and (29) into (30). By performing the integral, one gets

$$
\begin{aligned}
S= & \pi \Gamma\left(k+\frac{3}{2}\right) \\
& \times\left(\frac{r_{+}^{2}}{\gamma\left(k+3 / 2 ; r_{+}^{2} / 4 \theta\right)}-\frac{r_{e}^{2}}{\gamma\left(k+3 / 2 ; r_{e}^{2} / 4 \theta\right)}\right) \\
& +\pi \Gamma\left(k+\frac{3}{2}\right) \int_{r_{e}}^{r_{+}} d r r^{2} \frac{\gamma^{\prime}}{\gamma^{2}} .
\end{aligned}
$$

The first term can be written in terms of the area of the event horizon as

$$
\begin{aligned}
S= & \frac{1}{4 G_{N}} \Gamma\left(k+\frac{3}{2}\right) \\
& \times\left(\frac{A_{H}}{\gamma\left(k+3 / 2 ; r_{+}^{2} / 4 \theta\right)}-\frac{A_{e}}{\gamma\left(k+3 / 2 ; r_{e}^{2} / 4 \theta\right)}\right)+\cdots
\end{aligned}
$$

and represents the "area law" in our case. We have reinserted the Newton constant into (32) for reasons to become clear in a while. The standard form, which is one-fourth of the area, is recovered in the large black hole limit, $r_{H} \gg \sqrt{\theta}$.

Once (32) is written in natural units, we can define an effective Newton constant as

$$
G_{N} \longrightarrow G_{N}\left(r_{+}\right) \equiv G_{N} \frac{\gamma\left(k+3 / 2 ; r_{+}^{2} / 4 \theta\right)}{\Gamma(k+3 / 2)}
$$

and introduce a "modified" area law as

$$
S\left(r_{H}\right)=\frac{\pi r_{H}^{2}}{4 G_{N}\left(r_{H}\right)} .
$$

The interesting feature of $G_{N}\left(r_{H}\right)$ is to be "asymptotically free" in the sense that it is smaller than $G_{N}$, which represents the asymptotic value of the gravitational coupling for large black holes only (it is interesting to remark that if we apply the same reasoning to the Newton constant in (8), and replace $r_{H}$ with the radial coordinate $r$, we find a radial distancedependent gravitational coupling, which is quickly vanishing in the limit $r \rightarrow 0$. This asymptotically free behavior of our model, at short distance, provides an alternative explanation for the absence of curvature singularity in $r=0$ ).

Finally, the second term in (31) gives exponentially small corrections to the leading area term. It can be analytically computed for $k \gg 1$, but it does not introduce any relevant new effect.

\section{Conclusions}

In this paper, we derived new static, spherically symmetric regular solutions of Einstein's equations, describing the final state of collapsing matter shells in the presence of an effective minimal length. This derivation is the result of a long path starting with the quest of quantum gravity regularized black hole solutions [21, 29-40]. We showed that our previous discoveries of noncommutative geometry inspired solutions correspond to the simplest case within a larger class of regular gravitational objects. This class, the family of gravitational shells here presented, has peculiar properties which descend from the common key feature of the absence of curvature singularities. Such properties are the existence of extremal configurations, characterized by a minimal mass below which horizon cannot form, even in the case of neutral, nonrotating black holes; the occurrence of a phase transition from a thermodynamically unstable classical phase to a thermodynamically stable cooling down in the final stage of the horizon evaporation. As for the case of regular black holes, these matter shells correspond to final configurations of dynamical processes of gravitational collapse in which matter cannot be compressed below a fundamental length scale. The above properties are also common to other models of quantum geometry that have been derived by means of different formalisms. Specifically the shell profile of the energy density resembles what one has in the case of loop quantum black holes, with consequent thermodynamic similarities [47-50].

The study of these objects is far from being complete. We believe that these new solutions can have repercussions in a variety of fields. Here we just mention that these regular shells could affect the stability of the de Sitter space, at least during inflationary epochs [38] and they could lead to new insights into the physics of nuclear matter via the gauge/gravity duality paradigm as well [43].

\section{Acknowledgments}

This work has been supported by the project "Evaporation of microscopic black holes" under the Grant NI 1282/2-1 of the German Research Foundation (DFG), by the Helmholtz International Center for FAIR within the framework of the LOEWE program (Landesoffensive zur Entwicklung Wissenschaftlich-Ökonomischer Exzellenz) launched by the State of Hesse and in part by the European Cooperation in Science and Technology (COST) action MP0905 "Black Holes in a Violent Universe." Alessio Orlandi would like to thank the Frankfurt Institute for Advanced Studies (FIAS), Frankfurt am Main, Germany, for the initial period of working on this project. Piero Nicolini and Alessio Orlandi would like to thank the Perimeter Institute for Theoretical Physics, Waterloo, ON, Canada, for the kind hospitality during the final period of working on this project.

\section{References}

[1] A. Aurilia, R. S. Kissack, R. Mann, and E. Spallucci, "Relativistic bubble dynamics: from cosmic inflation to hadronic bags," Physical Review D, vol. 35, no. 10, pp. 2961-2975, 1987. 
[2] A. Aurilia, G. Denardo, F. Legovini, and E. Spallucci, "Vacuum tension effects on the evolution of domain walls in the early universe," Nuclear Physics B, vol. 252, pp. 523-537, 1985.

[3] S. K. Blau, E. I. Guendelman, and A. H. Guth, "Dynamics of false-vacuum bubbles," Physical Review D, vol. 35, no. 6, pp. 1747-1766, 1987.

[4] E. Farhi, A. H. Guth, and J. Guven, "Is it possible to create a universe in the laboratory by quantum tunneling?" Nuclear Physics B, vol. 339, no. 2, pp. 417-490, 1990.

[5] W. Israel, "Singular hypersurfaces and thin shells in general relativity," Il Nuovo Cimento B, vol. 44, no. 1, pp. 1-14, 1966.

[6] W. Israel, "Singular hypersurfaces and thinshells in general relativity," Il Nuovo Cimento B, vol. 48, no. 2, p. 463, 1967.

[7] S. Ansoldi, A. Aurilia, R. Balbinot, and E. Spallucci, "Classical and quantum shell dynamics, and vacuum decay," Classical and Quantum Gravity, vol. 14, no. 10, pp. 2727-2755, 1997.

[8] G. L. Alberghi, R. Casadio, and G. Venturi, "Effective action and thermodynamics of radiating shells in general relativity," Physical Review D, vol. 60, no. 12, Article ID 124018, 11 pages, 1999.

[9] S. Ansoldi, "WKB metastable quantum states of a de SitterReissner-Nordstrom dust shell," Classical and Quantum Gravity, vol. 19, no. 24, pp. 6321-6344, 2002.

[10] G. L. Alberghi, R. Casadio, and G. Venturi, “Thermodynamics for radiating shells in anti-de Sitter space-time," Physics Letters $B$, vol. 557, no. 1-2, pp. 7-11, 2003.

[11] G. L. Alberghi, R. Casadio, and D. Fazi, "Classical dynamics and stability of collapsing thick shells of matter," Classical and Quantum Gravity, vol. 23, no. 5, pp. 1493-1506, 2006.

[12] L. J. Garay, "Quantum gravity and minimum length," International Journal of Modern Physics A, vol. 10, no. 2, pp. 145-165, 1995.

[13] X. Calmet, M. Graesser, and S. D. H. Hsu, "Minimum length from quantum mechanics and classical general relativity," Physical Review Letters, vol. 93, no. 21, Article ID 211101, 2004.

[14] M. Fontanini, E. Spallucci, and T. Padmanabhan, "Zero-point length from string fluctuations," Physics Letters B, vol. 633, no. 4-5, pp. 627-630, 2006.

[15] A. Smailagic and E. Spallucci, "UV divergence-free QFT on noncommutative plane," Journal of Physics A, vol. 36, no. 39, pp. L517-L521, 2003.

[16] A. Smailagic and E. Spallucci, "Feynman path integral on the non-commutative plane," Journal of Physics A, vol. 36, no. 33, pp. L467-L471, 2003.

[17] A. Smailagic, E. Spallucci, and Lorentz invariance, "Lorentz invariance and unitarity in UV finite NCQFT," Journal of Physics A, vol. 37, pp. 1-10, 2004.

[18] A. Smailagic and E. Spallucci, "Lorentz invariance, unitarity and UV-finiteness of QFT on noncommutative spacetime," Journal of Physics A, vol. 37, no. 28, pp. 7169-7178, 2004.

[19] E. Spallucci, A. Smailagic, and P. Nicolini, "Trace anomaly on a quantum spacetime manifold," Physical Review D, vol. 73, no. 8 , Article ID 084004, 2006.

[20] M. Kober and P. Nicolini, "Minimal scales from an extended hilbert space," Classical and Quantum Gravity, vol. 27, no. 24, Article ID 245024, 2010.

[21] P. Nicolini, "Noncommutative black holes, the final appeal to quantum gravity: a review," International Journal of Modern Physics A, vol. 24, no. 7, pp. 1229-1308, 2009.
[22] R. Banerjee, B. R. Majhi, and S. Samanta, "Noncommutative black hole thermodynamics," Physical Review D, vol. 77, no. 12, Article ID 124035, 2008.

[23] R. Casadio and P. Nicolini, "The decay-time of non-commutative micro-black holes," Journal of High Energy Physics, vol. 2008, no. 11, article 072, 2008.

[24] Y. S. Myung, Y.-W. Kim, and Y.-J. Park, “Thermodynamics and evaporation of the noncommutative black hole," Journal of High Energy Physics, vol. 2007, no. 2, article 012, 2007.

[25] Y. S. Myung, Y.-W. Kim, and Y.-J. Park, "Quantum cooling evaporation process in regular black holes," Physics Letters B, vol. 656, no. 4-5, pp. 221-225, 2007.

[26] Y. S. Myung, Y. W. Kim, and Y. J. Park, "Thermodynamics of regular black hole," General Relativity and Gravitation, vol. 41, no. 5, pp. 1051-1067, 2009.

[27] R. Brustein, D. Gorbonos, and M. Hadad, "Wald's entropy is equal to a quarter of the horizon area in units of the effective gravitational coupling," Physical Review D, vol. 79, no. 4, Article ID 044025, 9 pages, 2009.

[28] P. Nicolini, "Entropic force, noncommutative gravity, and ungravity," Physical Review D, vol. 82, no. 4, Article ID 044030, 2010.

[29] P. Nicolini, "A model of radiating black hole in noncommutative geometry," Journal of Physics A, vol. 38, no. 39, pp. L631-L638, 2005.

[30] P. Nicolini, A. Smailagic, and E. Spallucci, "The fate of radiating black holes in noncommutative geometry," in Proceedings of the 13th General Conference of European Physical Society; Beyond Einstein, European Space Agency, November 2005.

[31] P. Nicolini, A. Smailagic, and E. Spallucci, "Noncommutative geometry inspired Schwarzschild black hole," Physics Letters B, vol. 632, no. 4, pp. 547-551, 2006.

[32] E. Spallucci and S. Ansoldi, "Regular black holes in UV selfcomplete quantum gravity," Physics Letters B, vol. 701, no. 4, pp. 471-474, 2011.

[33] S. Ansoldi, P. Nicolini, A. Smailagic, and E. Spallucci, "Noncommutative geometry inspired charged black holes," Physics Letters B, vol. 645, no. 2-3, pp. 261-266, 2007.

[34] E. Spallucci, A. Smailagic, and P. Nicolini, "Non-commutative geometry inspired higher-dimensional charged black holes," Physics Letters B, vol. 670, no. 4-5, pp. 449-454, 2009.

[35] P. Nicolini and E. Spallucci, "Noncommutative geometryinspired dirty black holes," Classical and Quantum Gravity, vol. 27, no. 1, Article ID 015010, 2010.

[36] A. Smailagic and E. Spallucci, “Kerrr' black hole: the lord of the string," Physics Letters B, vol. 688, no. 1, pp. 82-87, 2010.

[37] L. Modesto and P. Nicolini, "Charged rotating noncommutative black holes," Physical Review D, vol. 82, no. 10, Article ID 104035, 2010.

[38] R. B. Mann and P. Nicolini, "Cosmological production of noncommutative black holes," Physical Review D, vol. 84, no. 6, Article ID 064014, 2011.

[39] J. R. Mureika and P. Nicolini, "Aspects of noncommutative (1+ 1)-dimensional black holes," Physical Review D, vol. 84, no. 4, Article ID 044020, 2011.

[40] L. Modesto, J. W. Moffat, and P. Nicolini, "Black holes in an ultraviolet complete quantum gravity;" Physics Letters B, vol. 695, no. 1-4, pp. 397-400, 2011.

[41] P. Gaete, J. A. Helaÿel-Neto, and E. Spallucci, "Un-graviton corrections to the Schwarzschild black hole," Physics Letters B, vol. 693, no. 2, pp. 155-158, 2010. 
[42] J. R. Mureika and E. Spallucci, "Vector unparticle enhanced black holes: exact solutions and thermodynamics," Physics Letters B, vol. 693, no. 2, pp. 129-133, 2010.

[43] P. Nicolini and G. Torrieri, "The Hawking-Page crossover in noncommutative anti-deSitter space," Journal of High Energy Physics, vol. 2011, article 97, 2011.

[44] J. Mureika, P. Nicolini, and E. Spallucci, "Could any black holes be produced at the LHC?" Physical Review D, vol. 85, no. 10, Article ID 106007, 8 pages, 2012.

[45] J. J. Oha and C. Park, "Gravitational collapse of the shells with the smeared gravitational source in noncommutative geometry," Journal of High Energy Physics, vol. 2010, article 86, 2010.

[46] P. Nicolini and E. Winstanley, "Hawking emission from quantum gravity black holes," Journal of High Energy Physics, vol. 2011, article 75, 2011.

[47] L. Modesto, "Loop quantum black hole," Classical and Quantum Gravity, vol. 23, no. 18, pp. 5587-5601, 2006.

[48] L. Modesto and I. Premont-Schwarz, "Self-dual black holes in LQG: theory and phenomenology," Physical Review D, vol. 80, no. 6, Article ID 064041, 17 pages, 2009.

[49] S. Hossenfelder, L. Modesto, and I. Prémont-Schwarz, "Model for nonsingular black hole collapse and evaporation," Physical Review D, vol. 81, no. 4, Article ID 044036, 7 pages, 2010.

[50] L. Modesto, "Semiclassical loop quantum black hole," International Journal of Theoretical Physics, vol. 49, no. 8, pp.1649-1683, 2010. 

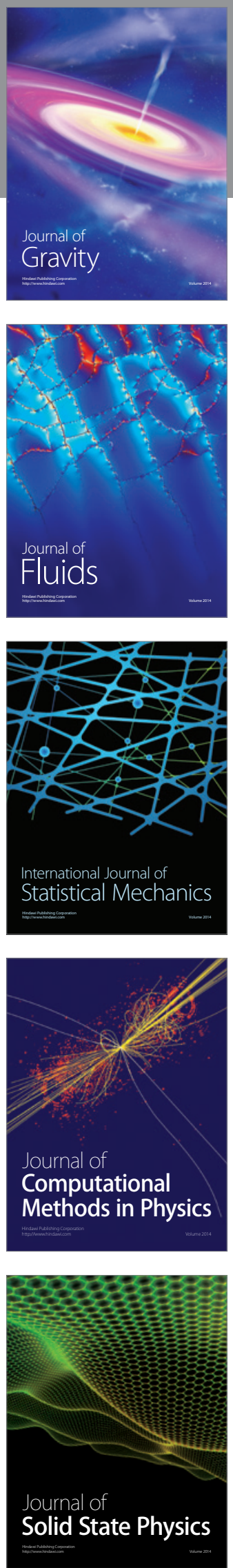

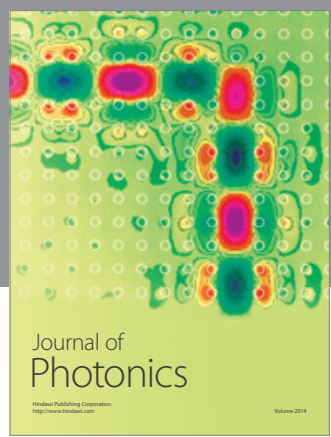

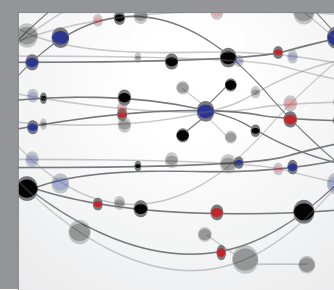

The Scientific World Journal

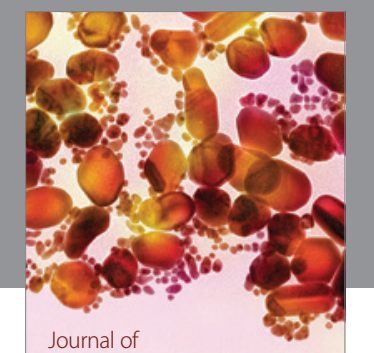

Soft Matter
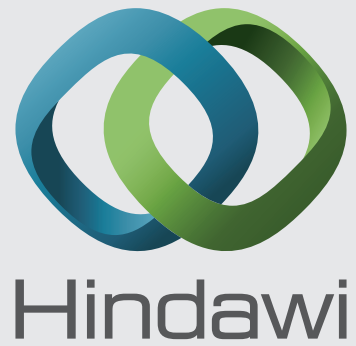

Submit your manuscripts at

http://www.hindawi.com
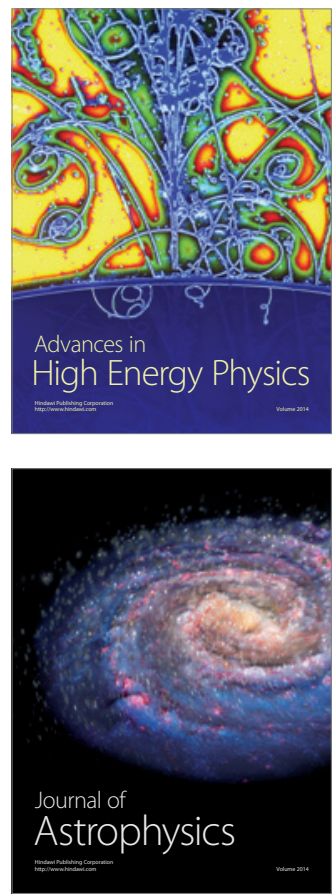
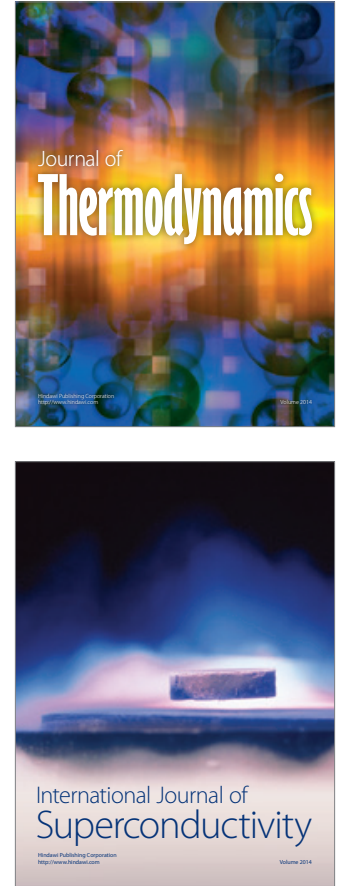
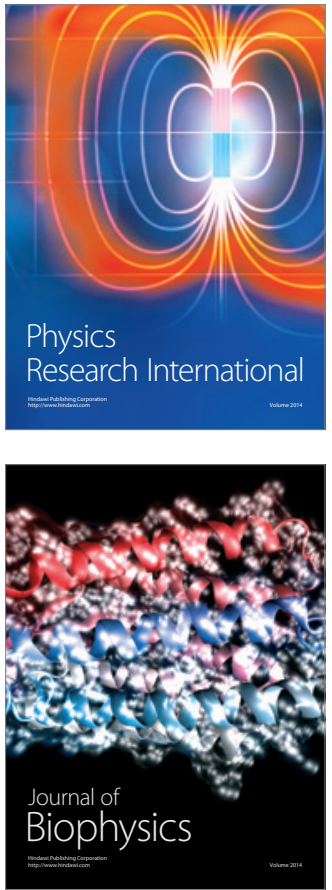
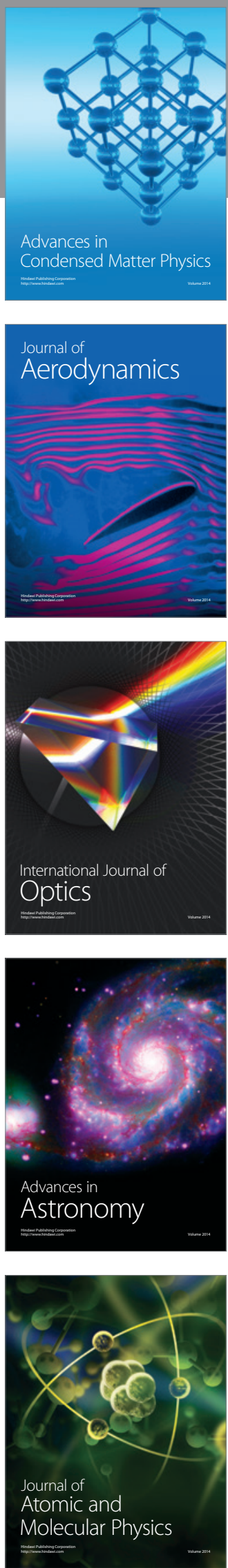